\title{
Behavioral Biases as An Effective Factor for the Firm Financial Decision- Making: A Literature Review
}

\author{
Bilgehan TEKIN \\ Cankiri Karatekin University, Department of Business Administration, Cankiri \\ btekin@karatekin.edu.tr
}

\begin{abstract}
Decision-making process is a multi-faceted and complex process. Decision making can be defined like a process of choosing from among a number of alternatives. It will not contribute enough to be fully understood and to effective decision making to be addressed only from the rational point of view. Behavioral finance is an integral part of the decisionmaking process. Individuals can improve their performance by recognizing the biases which discussed in the framework of behavioral finance. Understanding the possible negative effects of biases allows to the individuals to make better choices and they can avoid repeating the expensive errors in future. Result of investigations of behavioral biases on decisionmakers in the firms, managerial bias issue has been raised. The studies show the effect of managerial biases on many financial decisions in firms. This paper investigated the role of biases such as overconfidence, loss aversion, optimism, anchoring, narrow framing, self-serving attribution, disposition effect etc. on financial decisions such as investing, financing, equity market, capital structure etc. This study review of 30 international studies related with behavioral corporate finance and behavioral biases that affect financial decisions in firms. The studies were gleaned from Web of Science and Google Scholar. The main contribution of this study to the literature is this study brings out the impact of behavioral biases on financial decisions in the firms by summarizing the previous studies. In this sense, this work also has an assembly quality. Therefore, this is also intended with this study that to transfer the knowledge and intellectual formation about the impact of behavioral bias on the financial decisions. In this paper, most important behavioral biases in the behavioral finance literature will be addressed.
\end{abstract}

Keywords: Behavioral finance, behavioral biases, decision-making, managerial biases, financial decisions, corporate decisions.

\section{INTRODUCTION}

The questions such as; "what are the determinants of managerial decision-making processes?", "how is the decisionmaking process of managers?" and "how their psychological and characteristic features are affecting their decisions?" have not been answered fully by management and finance sciences, yet. As it is known, empirical and theoretical studies that are conducted in the field of economics, have a common assumption; "individuals are fully rational decision-makers". The basic theories of finance and management were derived on the basis of rational acting managers in terms of interests of shareholders, can create value by minimizing the potential effects of some factors such as taxes, bankruptcy costs, transaction costs, information asymmetries and diversified stakeholders. However, these theories have limited success in empirical tests (Adam, Fernando and Golubeva, 2015).

Behavioral finance, in general, investigates how the behavioral factors have an effect on the individuals' decision-making process. This psychological reality which is known as bias, in more broad terms, is deviations from rationality in the decision-making process, to repeat the perceptual distortion, unreasonable interpretation and flawed judgment (Bashir et al., 2013).

The lack of knowledge leads to contain uncertainty of the majority of financial decisions that made in company. Because of individuals make decision under uncertainty and exhibit continuously irrational behavior in a systematic way stems from psychological factors such as cognitive bias, intuition and emotion (Camerer, 2004; Kahneman, 1979). Irrational decisions will be cause of taking seriously wrong decisions and detrimental to the firm value. This phenomenon is called "behavioral cost" and represents negative relationship between firm value and irrational behavior (Shefrin, 2001).

Kahneman and Tversky's (1979) findings of their study on financial decision making under uncertainty, shows that individuals behave not as specified in the traditional models. In question study, also include a critique of expected utility theory and suggest an alternative model as a result of this critique. This model is "prospect theory". Prospect Theory state that despite the individuals take risk to avoid losses, when earnings are concerned they are risk averse. Therefore, the basis of their decisions is perception of earnings. The behavioral or cognitive bias is "intuitive preferences that consistently violets the rules of rational choice" according to Kahneman (2011). When a psychological pattern change a person's subjective view of an issue to differ from reality this case shows that there is an effect of bias on decision making.

In 1985, Sturdivant, Ginter and Sawyer examined the conservatism bias phenomenon basis on managers' and corporate performance. They developed a scale to measure conservatism. The results of the study showed that conservative bias among the senior management groups was a negative relationship with the firm's social responsibility in addition to certain aspects of the financial performance (Sturdivant et al., 1985).

Roll, in his study in 1986, express that many evidence about the acquisition operations, indicates that it is compatible with the economy in the absence of the gain related to the acquisition, but in the situation of managers are overconfident, this is Roll's "hubris hypothesis". When prompted to take over a company, managers take into account the synergies which will 


\section{ISSN $2321-1091$ \\ Volume $10 \mathrm{Number} 2$ \\ Journal of Social Sciences Research}

be occur in the future, as well as to the valuation analysis. The overconfidence that owned by managers about the accuracy of the analysis made by them, will encourage them to act now when exceeds the amount of the valuation to market prices (Barberis and Thaler, 2003).

As Baker and Wurgler (2007) are mentioned in their study, behavioral finance and corporate finance researches provides displacement of traditional rationality assumptions with the relatively more evidence-based behavioral basics. Behavioral finance examines the biases that managers, investors and other market participants exhibit when they make a financia decision. Behavioral biases can be expressed in various forms: overconfidence, loss aversion, anchoring, self-serving attribution, optimism etc. Every behavioral bias has a different effect on firm financial decision making.

In this study we examine the behavioral biases which affect the managerial decisions. The study focuses on and considers the most important studies and biases in the behavioral finance literature about the managerial biases. This study, in its nature, a review of the literature of the most examined behavioral biases' which affect the managers' decisions. In order to get a deeper understanding of the subject, the references have tried to remain faithful to the original text and in most places words are not studied to be replaced. Another reason for the emergence of this study is the lack of literature on the subject in Turkey. Indeed, it is observed that behavioral corporate finance or administrative prejudices have little or no work considering the issue of the number of field work.

\section{LITERATURE REVIEW METHOD AND IDENTIFIED PUBLICATIONS}

This study reports the much-needed literature and findings of studies on the subject of behavioral bias and its impact on the firm and managers' decisions. For this purpose, Google Scholar and the Web of Science (WOS) databases were scanned with the terms of, in quotation marks, "behavioral finance", "behavioral corporate finance", "behavioral biases", "managerial bias", "psychological bias", "financial decisions" and "corporate/firm/company decisions" through together and separately. The search that made on WOS, includes the year of between 1975 and 2015. Model of the search made on WOS is given schematically as follows in Figure 1.

The applied WOS filters before scanning are respectively as follows; primarily basic research was done by entering the term of "behavioral finance" to the topic. As shown in Table 1, as a result of this initial search has reached a total of 705 publications. Later research domains "Social Sciences", research area "Business Economics" and languages "English" filters were applied respectively. As a result of this search has reached a total of 474 publications. Then continue to search with other terms by using the same filters. The reached publication numbers as a result of scans with other words are as follows: "behavioral corporate finance" 19, "behavioral bias" 109, "managerial bias" 8, "psychological bias" 26, "financial decision" 282 and "corporate/firm/company decision" 157 (Table 1).

Table 1. Search results by topics \& filters

\begin{tabular}{|c|c|c|}
\hline TOPICS & Initial Search Result & $\begin{array}{c}\text { Number of Publications after } \\
\text { Filters }\end{array}$ \\
\hline Behavioral Finance & 705 & 474 \\
\hline Behavioral Corporate Finance & & 19 \\
\hline Behavioral Biases & & 109 \\
\hline Managerial Bias & & 26 \\
\hline Psychological Bias & & 282 \\
\hline Financial Decision & & 157 \\
\hline
\end{tabular}

13 out of these publications were included to this study, and examined. These 13 publications are determined based on the number of citations, kind of behavioral biases, financial decisions and delivery dates (relatively current). Bradford (1934) indicates that must taken into account the studies that published in which called basic or core journal and most interest with the subject, in bibliometric studies. This phenomenon is known as "Bradford Law". Bradford Law is based on the principle that ranking periodicals in the selected literature, according to the degree of efficiency (Gökkurt, 1994). 


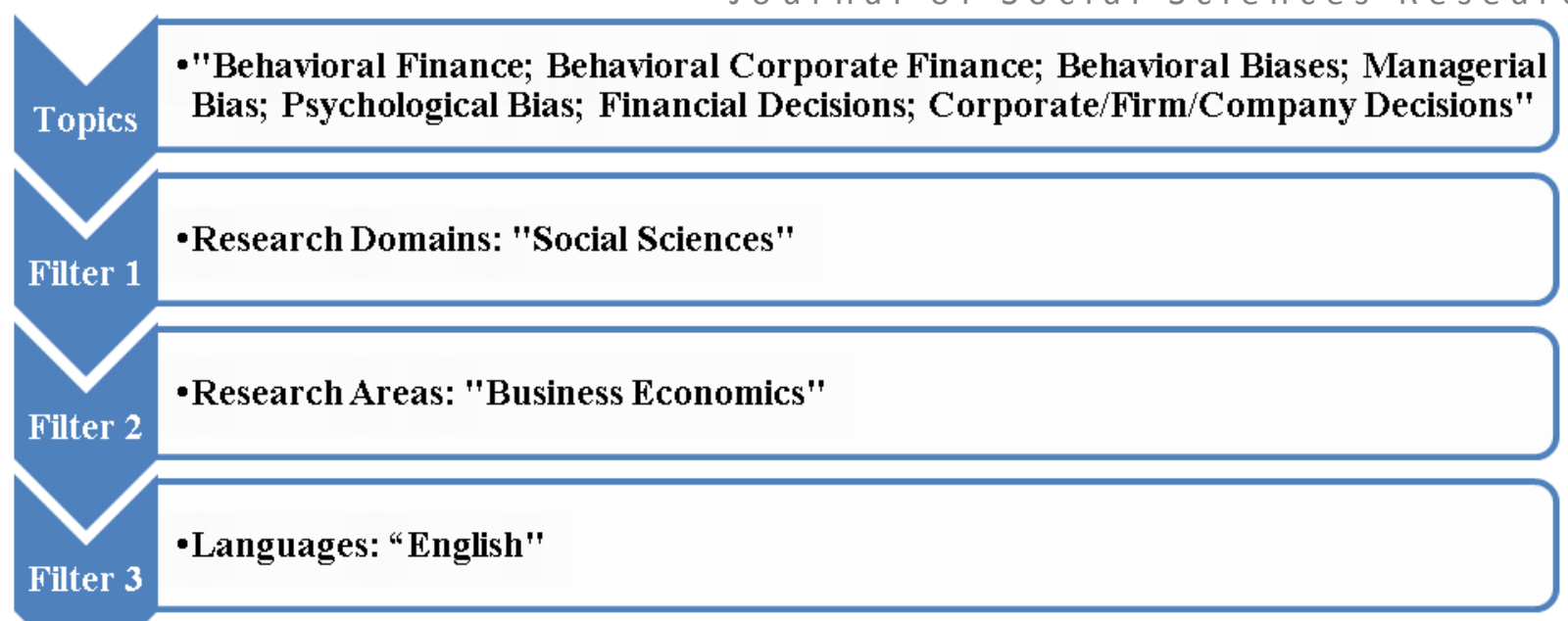

Fig 1: Search Model Made on WOS

The same terms were searched in Google scholar and a total of 30 publications (13 were also be scanned in WOS) were evaluated according to the same criteria. Consequently, the information about the retrieved publications is shown in the Table 1 as a summary and in chronological order. Now those studies will be discussed in a little more detail. For example, Burgstahler \& Dichev (1997) indicate, the firms manage reported earnings to avoid earnings decreases and losses. This is a kind of examining the phenomenon of loss aversion. They present two theories, based on stakeholder use of information-processing heuristics and prospect theory, about the motivation for avoidance of earnings decreases and losses.

Bernardo and Welch (2001) have examined that why seemingly irrational overconfident behavior can persist. They offer an explanation of overconfidence and entrepreneurs. The authors stated that overconfident entrepreneurs less imitate the environment then peers and they are more likely to explore their environment.

Malmendier and Tate (2001) have examined if the over-optimistic managers show more sensitive to investments cash flows. The authors investigated the use of stock options of managers and they reached the conclusion that the managers are excessively optimistic. They also find that the firms which are managed by over-optimistic managers, are more susceptible to cash flow of investments (Barberis and Thaler, 2003). In another version of their study, Malmendier and Tate (2005) state that overconfidence affects corporate policies such as capital expenditures and merger and acquisitions (Malmendier and Tate, 2008), financing decisions (Malmendier et al., 2011). Heaton (2002) has addressed managerial irrationality under a single dimension with optimism bias. Heaton express that optimism bias can lead to the perception of company's stock prices miss valued by the stock market. Therefore, investment projects with net present value can be ignored for the reason that it requires external source financing. On the other hand, optimism can lead to value of negative net present value projects as exaggerated, so it is possible that investing to this type of projects by optimistic managers. 
Journal of Social Sciences Research

Table 2. Literature review of studies about behavioral bias and its impact on the decisions of firm and managers

\begin{tabular}{|c|c|c|c|c|c|c|c|c|}
\hline \multirow[t]{2}{*}{ Author (s) } & \multirow[t]{2}{*}{ Title } & \multirow{2}{*}{$\begin{array}{c}\text { Behavioral } \\
\text { Bias }\end{array}$} & \multirow[t]{2}{*}{ Topic } & \multirow{2}{*}{$\begin{array}{c}\text { Journal/ } \\
\text { Publishe } \\
r\end{array}$} & \multirow{2}{*}{$\begin{array}{c}\text { Publica } \\
\text { tion } \\
\text { Type }\end{array}$} & \multirow[t]{2}{*}{ Year } & \multicolumn{2}{|c|}{ Citation $^{\star}$} \\
\hline & & & & & & & $\mathrm{GS}_{1}$ & $\begin{array}{l}\text { WO } \\
S^{2}\end{array}$ \\
\hline $\begin{array}{l}\text { Burgstahle } \\
\text { r, D., \& } \\
\text { Dichev, I. }\end{array}$ & $\begin{array}{l}\text { Earnings } \\
\text { managem } \\
\text { ent to } \\
\text { avoid } \\
\text { earnings } \\
\text { decreases } \\
\text { and } \\
\text { losses. }\end{array}$ & Loss Aversion & $\begin{array}{l}\text { Earning } \\
\text { decreases \& } \\
\text { losses. }\end{array}$ & $\begin{array}{l}\text { Journal } \\
\text { of } \\
\text { accountin } \\
\text { g \& \& } \\
\text { economic } \\
\text { s }\end{array}$ & Article & 1997 & $\begin{array}{l}32 \\
69\end{array}$ & 550 \\
\hline $\begin{array}{l}\text { Bernardo, } \\
\text { A. E., \& } \\
\text { Welch, I. }\end{array}$ & $\begin{array}{l}\text { On the } \\
\text { evolution } \\
\text { of } \\
\text { overconfid } \\
\text { ence and } \\
\text { entreprene } \\
\text { urs. }\end{array}$ & $\begin{array}{l}\text { Overconfidenc } \\
\mathrm{e}\end{array}$ & $\begin{array}{l}\text { Entrepreneur } \\
\text { s' imitate \& } \\
\text { explore } \\
\text { behavior }\end{array}$ & $\begin{array}{l}\text { Journal } \\
\text { of } \\
\text { economic } \\
\text { s \& } \\
\text { manage } \\
\text { ment } \\
\text { strategy }\end{array}$ & Article & 2001 & $\begin{array}{l}44 \\
5\end{array}$ & 99 \\
\hline $\begin{array}{l}\text { Malmendie } \\
\text { r, U., \& } \\
\text { Tate, G. }\end{array}$ & $\begin{array}{l}\text { CEO } \\
\text { overconfid } \\
\text { ence and } \\
\text { corporate } \\
\text { investment }\end{array}$ & $\begin{array}{l}\text { Overconfidenc } \\
\text { e }\end{array}$ & $\begin{array}{l}\text { Corporate } \\
\text { investment } \\
\text { distortions }\end{array}$ & $\begin{array}{l}\text { The } \\
\text { journal of } \\
\text { finance }\end{array}$ & Article & $\begin{array}{l}2001 / 2 \\
005\end{array}$ & $\begin{array}{l}20 \\
87\end{array}$ & 373 \\
\hline $\begin{array}{l}\text { Heaton, J. } \\
\text { B. }\end{array}$ & $\begin{array}{l}\text { Managerial } \\
\text { optimism } \\
\text { and } \\
\text { corporate } \\
\text { finance. }\end{array}$ & Optimism & $\begin{array}{l}\text { Free cash } \\
\text { flows }\end{array}$ & $\begin{array}{l}\text { Financial } \\
\text { manage } \\
\text { ment }\end{array}$ & Article & 2002 & $\begin{array}{l}92 \\
1\end{array}$ & 121 \\
\hline $\begin{array}{l}\text { Willman, } \\
\text { P., } \\
\text { Fenton- } \\
\text { O'Creevy, } \\
\text { M., } \\
\text { Nicholson, } \\
\text { N., \& } \\
\text { Soane, E. }\end{array}$ & $\begin{array}{l}\text { Traders, } \\
\text { managers } \\
\text { and loss } \\
\text { aversion in } \\
\text { investment } \\
\text { banking: a } \\
\text { field study }\end{array}$ & Loss Aversion & $\begin{array}{l}\text { The } \\
\text { Management } \\
\text { of Traders }\end{array}$ & $\begin{array}{l}\text { Accounti } \\
\text { ng, } \\
\text { organizati } \\
\text { ons \& } \\
\text { society }\end{array}$ & Article & 2002 & 40 & 12 \\
\hline Barberis, & A survey & Rational & Limits & Handboo & Article & 2003 & 28 & - \\
\hline
\end{tabular}


Journal of Social Sciences Research

\begin{tabular}{|c|c|c|c|c|c|c|c|c|}
\hline $\begin{array}{l}\text { N., \& } \\
\text { Thaler, R }\end{array}$ & $\begin{array}{l}\text { of } \\
\text { behavioral } \\
\text { finance }\end{array}$ & $\begin{array}{l}\text { Investors \& } \\
\text { Irrational } \\
\text { Managers/Rat } \\
\text { ional } \\
\text { Managers and } \\
\text { Irrational } \\
\text { Investors } \\
\end{array}$ & $\begin{array}{l}\text { Arbitrage \& } \\
\text { Psychology }\end{array}$ & $\begin{array}{l}\mathrm{k} \text { of the } \\
\text { Economi } \\
\mathrm{cs} \text { of } \\
\text { Finance }\end{array}$ & $\begin{array}{l}\text { (Chapte } \\
r \quad \text { of } \\
\text { Book) }\end{array}$ & & 13 & \\
\hline Shefrin, $\mathrm{H}$ & $\begin{array}{l}\text { A } \\
\text { Behavioral } \\
\text { Approach } \\
\text { to Asset } \\
\text { Pricing }\end{array}$ & $\begin{array}{l}\text { Representativ } \\
\text { eness, } \\
\text { Overconfidenc } \\
\text { e, Availability } \\
\text { Bias } \\
\text { Optimism/Pes } \\
\text { simism } \\
\text { Risk } \\
\text { Aversion/Loss } \\
\text { Aversion, } \\
\text { Status Quo, } \\
\text { Framing, } \\
\text { Ambiguity }\end{array}$ & Asset Pricing & $\begin{array}{l}\text { Boston: } \\
\text { Elsevier } \\
\text { Academi } \\
\text { c Press. }\end{array}$ & Book & $\begin{array}{l}2005 / 2 \\
008\end{array}$ & $\begin{array}{l}43 \\
4\end{array}$ & - \\
\hline $\begin{array}{l}\text { Ljungqvist, } \\
\text { A., \& } \\
\text { Wilhelm, } \\
\text { W. J. }\end{array}$ & $\begin{array}{l}\text { Does } \\
\text { prospect } \\
\text { theory } \\
\text { explain } \\
\text { IPO } \\
\text { market } \\
\text { behavior? }\end{array}$ & $\begin{array}{l}\text { Prospect } \\
\text { Theory } \\
\text { Anchoring/Ref } \\
\text { erence Point/ } \\
\text { Risk } \\
\text { Aversion/Loss } \\
\text { Aversion }\end{array}$ & $\begin{array}{l}\text { IPO } \\
\text { Performance }\end{array}$ & $\begin{array}{l}\text { The } \\
\text { Journal } \\
\text { of } \\
\text { Finance }\end{array}$ & Article & 2005 & $\begin{array}{l}15 \\
8\end{array}$ & 28 \\
\hline $\begin{array}{c}\text { Barberis, } \\
\text { N., } \\
\& \\
\mathrm{Hu} \\
\text { ang } \\
\text {, M. }\end{array}$ & $\begin{array}{l}\text { The loss } \\
\text { aversion/ } \mathrm{N} \\
\text { arrow } \\
\text { framing } \\
\text { approach } \\
\text { to the } \\
\text { equity } \\
\text { premium } \\
\text { puzzle }\end{array}$ & $\begin{array}{l}\text { Loss Aversion } \\
\text { Narrow } \\
\text { Framing }\end{array}$ & Equity Puzzle & $\begin{array}{l}\text { National } \\
\text { Bureau of } \\
\text { Economi } \\
\text { C } \\
\text { Research }\end{array}$ & Article & 2006 & 79 & - \\
\hline $\begin{array}{l}\text { Dodonova, } \\
\text { A., \& } \\
\text { Khoroshilo } \\
\text { v, Y. }\end{array}$ & $\begin{array}{l}\text { Jump } \\
\text { bidding in } \\
\text { takeover } \\
\text { auctions }\end{array}$ & $\begin{array}{l}\text { Loss } \\
\text { Aversion/Risk } \\
\text { aversion }\end{array}$ & $\begin{array}{l}\text { Takeover } \\
\text { auctions }\end{array}$ & $\begin{array}{l}\text { Economi } \\
\text { cs Letters }\end{array}$ & Article & 2006 & 20 & 5 \\
\hline $\begin{array}{l}\text { Ben- } \\
\text { David, I., }\end{array}$ & $\begin{array}{l}\text { Managerial } \\
\text { overconfid }\end{array}$ & $\begin{array}{l}\text { Overconfidenc } \\
\mathrm{e}\end{array}$ & $\begin{array}{l}\text { Corporate } \\
\text { Policies: }\end{array}$ & $\begin{array}{l}\text { National } \\
\text { Bureau of }\end{array}$ & Article & 2007 & $\begin{array}{l}34 \\
7\end{array}$ & - \\
\hline
\end{tabular}




\begin{tabular}{|c|c|c|c|c|c|c|c|c|}
\hline $\begin{array}{l}\text { Graham, } \\
\text { J. R., \& } \\
\text { Harvey, C. } \\
\text { R. }\end{array}$ & $\begin{array}{l}\text { ence \& } \\
\text { corporate } \\
\text { policies }\end{array}$ & & $\begin{array}{l}\text { investment, } \\
\text { financing, } \\
\text { reporting, \& } \\
\text { executive } \\
\text { compensatio } \\
\mathrm{n}\end{array}$ & $\begin{array}{l}\text { Economi } \\
\mathrm{c} \\
\text { Research }\end{array}$ & & & & \\
\hline $\begin{array}{l}\text { Devers, C. } \\
\text { E., } \\
\text { Wiseman, } \\
\text { R. M., \& } \\
\text { Holmes, } \\
\text { R. M. }\end{array}$ & $\begin{array}{l}\text { The effects } \\
\text { of } \\
\text { endowmen } \\
\mathrm{t} \& \text { loss } \\
\text { aversion in } \\
\text { managerial } \\
\text { stock } \\
\text { option } \\
\text { valuation }\end{array}$ & $\begin{array}{l}\text { Endowment \& } \\
\text { Loss Aversion }\end{array}$ & $\begin{array}{l}\text { Stock Option } \\
\text { Valuation }\end{array}$ & $\begin{array}{l}\text { Academy } \\
\text { of } \\
\text { Manage } \\
\text { ment } \\
\text { Journal }\end{array}$ & Article & 2007 & 81 & 35 \\
\hline $\begin{array}{r}\text { Brown, R., } \\
\& \\
\text { Sar } \\
\text { ma, } \\
\text { N.. }\end{array}$ & $\begin{array}{l}\text { CEO } \\
\text { overconfid } \\
\text { ence, CEO } \\
\text { dominance } \\
\& \\
\text { corporate } \\
\text { acquisition } \\
\text { s }\end{array}$ & $\begin{array}{l}\text { Overconfidenc } \\
\text { e }\end{array}$ & Acquisitions & $\begin{array}{l}\text { Journal } \\
\text { of } \\
\text { Economi } \\
\text { cs and } \\
\text { business, }\end{array}$ & Article & 2007 & $\begin{array}{l}16 \\
8\end{array}$ & - \\
\hline $\begin{array}{l}\text { Campbell, } \\
\text { S. D., \& } \\
\text { Sharpe, S. } \\
\text { A. }\end{array}$ & $\begin{array}{l}\text { Anchoring } \\
\text { bias in } \\
\text { consensus } \\
\text { forecasts } \\
\text { \& its effect } \\
\text { on market } \\
\text { prices }\end{array}$ & Anchoring & $\begin{array}{l}\text { Market } \\
\text { Prices of } \\
\text { Securities }\end{array}$ & $\begin{array}{l}\text { Journal } \\
\text { of } \\
\text { Financial } \\
\& \\
\text { Quantitati } \\
\text { ve } \\
\text { Analysis }\end{array}$ & Article & 2009 & 85 & 13 \\
\hline $\begin{array}{r}\text { Fairchild, } \\
\text { R. }\end{array}$ & $\begin{array}{l}\text { Managerial } \\
\text { overconfid } \\
\text { ence, } \\
\text { moral } \\
\text { hazard } \\
\text { problems, } \\
\text { and } \\
\text { excessive } \\
\text { life cycle } \\
\text { debt } \\
\text { sensitivity }\end{array}$ & $\begin{array}{l}\text { Overconfidenc } \\
\text { e }\end{array}$ & $\begin{array}{l}\text { Financing } \\
\text { Decisions \& } \\
\text { Firm Value }\end{array}$ & $\begin{array}{l}\text { Investme } \\
\text { nt } \\
\text { Manage } \\
\text { ment and } \\
\text { Financial } \\
\text { Innovatio } \\
\text { ns }\end{array}$ & Article & 2009 & 8 & - \\
\hline Hribar, P., & CEO & Overconfidenc & Management & Contemp & Article & 2015 & 73 & - \\
\hline
\end{tabular}




\begin{tabular}{|c|c|c|c|c|c|c|c|c|}
\hline $\begin{array}{l}\text { \& } \\
\text { Ya } \\
\text { ng, } \\
\text { H. }\end{array}$ & $\begin{array}{l}\text { overconfid } \\
\text { ence and } \\
\text { managem } \\
\text { ent } \\
\text { forecasting }\end{array}$ & $\mathrm{e}$ & $\begin{array}{l}\text { earnings } \\
\text { forecasts }\end{array}$ & $\begin{array}{l}\text { orary } \\
\text { Accounti } \\
\text { ng } \\
\text { Research }\end{array}$ & & & & \\
\hline Li, F. & $\begin{array}{l}\text { Managers' } \\
\text { self- } \\
\text { serving } \\
\text { attribution } \\
\text { bias \& } \\
\text { corporate } \\
\text { financial } \\
\text { policies. }\end{array}$ & $\begin{array}{l}\text { Self-serving } \\
\text { attribution }\end{array}$ & $\begin{array}{l}\text { Corporate } \\
\text { Policies }\end{array}$ & SSRN & Article & 2010 & 19 & - \\
\hline $\begin{array}{l}\text { Galasso, } \\
\text { A., \& } \\
\text { Simcoe, T. } \\
\text { S }\end{array}$ & $\begin{array}{l}\text { CEO } \\
\text { overconfid } \\
\text { ence \& } \\
\text { innovation }\end{array}$ & $\begin{array}{l}\text { Overconfidenc } \\
\mathrm{e}\end{array}$ & Innovation & $\begin{array}{l}\text { Manage } \\
\text { ment } \\
\text { Science }\end{array}$ & Article & 2011 & $\begin{array}{l}17 \\
6\end{array}$ & 27 \\
\hline $\begin{array}{l}\text { Huang, C. } \\
\text { H., \& } \\
\text { Padmanab } \\
\text { han, P. }\end{array}$ & $\begin{array}{l}\text { Off- } \\
\text { Shoring, } \\
\text { Managerial } \\
\text { Anchoring, } \\
\text { \& Firm } \\
\text { Value: A } \\
\text { Two } \\
\text { Period } \\
\text { Model. }\end{array}$ & Anchoring & Firm Value & $\begin{array}{l}\text { Internatio } \\
\text { nal } \\
\text { Review } \\
\text { of } \\
\text { Business } \\
\text { Research } \\
\text { Papers }\end{array}$ & Article & 2011 & 1 & - \\
\hline $\begin{array}{l}\text { Libby, R., } \\
\text { \& } \\
\text { Rennekam } \\
\text { p, K. }\end{array}$ & $\begin{array}{l}\text { Self-Servin } \\
\text { g } \\
\text { Attribution } \\
\text { Bias, } \\
\text { Overconfid } \\
\text { ence, \& } \\
\text { the } \\
\text { Issuance } \\
\text { of } \\
\text { Managem } \\
\text { ent } \\
\text { Forecasts. }\end{array}$ & $\begin{array}{l}\text { Self-Serving } \\
\text { Attribution, } \\
\text { Overconfidenc } \\
\mathrm{e}\end{array}$ & $\begin{array}{l}\text { Firm } \\
\text { Performance } \\
\text { Management } \\
\text { Forecasts }\end{array}$ & $\begin{array}{l}\text { Journal } \\
\text { of } \\
\text { Accounti } \\
\text { ng } \\
\text { Research }\end{array}$ & Article & 2012 & 59 & 6 \\
\hline $\begin{array}{l}\text { Baker, M., } \\
\text { Pan, X., \& } \\
\text { Wurgler, J. }\end{array}$ & $\begin{array}{l}\text { The effect } \\
\text { of } \\
\text { reference } \\
\text { point }\end{array}$ & $\begin{array}{l}\text { Reference } \\
\text { Point/Anchori } \\
\text { ng }\end{array}$ & $\begin{array}{l}\text { Mergers and } \\
\text { Acquisitions }\end{array}$ & $\begin{array}{l}\text { Journal } \\
\text { of } \\
\text { Financial } \\
\text { Economi }\end{array}$ & Article & 2012 & $\begin{array}{l}13 \\
7\end{array}$ & 18 \\
\hline
\end{tabular}




\begin{tabular}{|c|c|c|c|c|c|c|c|c|}
\hline & $\begin{array}{l}\text { prices on } \\
\text { mergers \& } \\
\text { acquisition } \\
\mathrm{s}\end{array}$ & & & CS & & & & \\
\hline $\begin{array}{l}\text { Ammann, } \\
\text { M., Ising, } \\
\text { A., \& } \\
\text { Kessler, S. }\end{array}$ & $\begin{array}{l}\text { Disposition } \\
\text { effect \& } \\
\text { mutual } \\
\text { fund } \\
\text { performan } \\
\text { ce }\end{array}$ & $\begin{array}{l}\text { Disposition } \\
\text { effect }\end{array}$ & Mutual Funds & $\begin{array}{l}\text { Applied } \\
\text { Financial } \\
\text { Economi } \\
\text { cs }\end{array}$ & Article & 2012 & 12 & - \\
\hline $\begin{array}{l}\text { Jackson, } \\
\text { S. B., } \\
\text { Keune, T. } \\
\text { M., \& } \\
\text { Salzsieder } \\
\text {, L. }\end{array}$ & $\begin{array}{l}\text { Debt, } \\
\text { equity, \& } \\
\text { capital } \\
\text { investment }\end{array}$ & $\begin{array}{l}\text { Mental } \\
\text { Accounting }\end{array}$ & $\begin{array}{l}\text { Capital } \\
\text { Structure/Bu } \\
\text { dgeting }\end{array}$ & $\begin{array}{l}\text { Journal } \\
\text { of } \\
\text { Accounti } \\
\text { ng \& \& } \\
\text { Economi } \\
\text { cs }\end{array}$ & Article & 2013 & 5 & 2 \\
\hline $\begin{array}{l}\text { Andriosop } \\
\text { oulos, D., } \\
\text { Andriosop } \\
\text { oulos, K., } \\
\text { \& Hoque, } \\
\text { H. }\end{array}$ & $\begin{array}{l}\text { Informatio } \\
\mathrm{n} \\
\text { disclosure, } \\
\text { CEO } \\
\text { overconfid } \\
\text { ence, \& } \\
\text { share } \\
\text { buyback } \\
\text { completion } \\
\text { rates }\end{array}$ & $\begin{array}{l}\text { Overconfidenc } \\
\text { e }\end{array}$ & $\begin{array}{l}\text { Share } \\
\text { buyback }\end{array}$ & $\begin{array}{l}\text { Journal } \\
\text { of } \\
\text { Banking } \\
\& \\
\text { Finance }\end{array}$ & Article & 2013 & 10 & - \\
\hline $\begin{array}{l}\text { Deshmukh } \\
\text {, S., Goel, } \\
\text { A. M., \& } \\
\text { Howe, K. } \\
\text { M. }\end{array}$ & $\begin{array}{l}\text { CEO } \\
\text { overconfid } \\
\text { ence \& } \\
\text { dividend } \\
\text { policy }\end{array}$ & $\begin{array}{l}\text { Overconfidenc } \\
\text { e }\end{array}$ & Dividend & $\begin{array}{l}\text { Journal } \\
\text { of } \\
\text { Financial } \\
\text { Intermedi } \\
\text { ation }\end{array}$ & Article & 2013 & 95 & - \\
\hline $\begin{array}{l}\text { Cen, L., } \\
\text { Hilary, G., } \\
\text { \& Wei, K. } \\
\text { C. }\end{array}$ & $\begin{array}{l}\text { The role of } \\
\text { anchoring } \\
\text { bias in the } \\
\text { equity } \\
\text { market: } \\
\text { Evidence } \\
\text { from } \\
\text { analysts' } \\
\text { earnings } \\
\text { forecasts } \\
\& \text { stock }\end{array}$ & Anchoring & $\begin{array}{l}\text { Equity } \\
\text { Market }\end{array}$ & $\begin{array}{l}\text { Journal } \\
\text { of } \\
\text { Financial } \\
\& \\
\text { Quantitati } \\
\text { ve } \\
\text { Analysis }\end{array}$ & Article & 2013 & 53 & - \\
\hline
\end{tabular}


Journal of Social Sciences Research

\begin{tabular}{|c|c|c|c|c|c|c|c|c|}
\hline & returns & & & & & & & \\
\hline $\begin{array}{l}\text { Adam, T., } \\
\text { Burg, V., } \\
\text { Scheinert, } \\
\text { T. \& } \\
\text { Streitz, D. }\end{array}$ & $\begin{array}{l}\text { Managerial } \\
\text { Optimism } \\
\text { \& Debt } \\
\text { Contract } \\
\text { Design: } \\
\text { The Case } \\
\text { of } \\
\text { Syndicated } \\
\text { Loans }\end{array}$ & Optimism & $\begin{array}{l}\text { Syndicated } \\
\text { Loan } \\
\text { Contracts }\end{array}$ & SSRN & Article & 2014 & 6 & - \\
\hline $\begin{array}{l}\text { Blanc, S. } \\
\text { M., } \quad \text { \& } \\
\text { Setzer, T. }\end{array}$ & $\begin{array}{l}\text { Analytical } \\
\text { debiasing } \\
\text { of } \\
\text { corporate } \\
\text { cash flow } \\
\text { forecasts }\end{array}$ & $\begin{array}{l}\text { Debiasing/For } \\
\text { ecast Bias }\end{array}$ & $\begin{array}{l}\text { Corporate } \\
\text { Cash Flow }\end{array}$ & $\begin{array}{l}\text { European } \\
\text { Journal } \\
\text { of } \\
\text { Operatio } \\
\text { nal } \\
\text { Research }\end{array}$ & Article & 2015 & 3 & - \\
\hline $\begin{array}{l}\text { Adam, T. } \\
\text { R., } \\
\text { Fernando, } \\
\text { C. S., \& } \\
\text { Golubeva, } \\
\text { E. }\end{array}$ & $\begin{array}{l}\text { Managerial } \\
\text { overconfid } \\
\text { ence and } \\
\text { corporate } \\
\text { risk } \\
\text { managem } \\
\text { ent }\end{array}$ & $\begin{array}{l}\text { Overconfidenc } \\
\mathrm{e}\end{array}$ & $\begin{array}{l}\text { Risk } \\
\text { Management }\end{array}$ & $\begin{array}{l}\text { Journal } \\
\text { of } \\
\text { Banking } \\
\& \\
\text { Finance }\end{array}$ & Article & 2015 & 7 & - \\
\hline
\end{tabular}


Willman, O' Creevy, Nicholson and Soane (2002) have discussed the management of traders in the financial markets in terms of prospect and agency theories. In their study, they examine the cases to achieve gains while avoiding losses. Managers, in the process of they are losing money they are entered into a closer relationship with traders. And also they indicate managers have such framing effects in a widespread.

Barberis and Thaler (2003) have indicated that the field of behavioral finance has two building blocks, limits to arbitrage and psychology. In their research they present a number of behavioral finance applications that relate with the aggregate stock market, the cross-section of average returns, individual trading behavior, and about the corporate finance. The authors also examine the irrational investors can affect the corporate financing and investment decisions. They express that it can be possible in some situations. They indicate there is some evidence that irrational investor's sentiment affects financing decisions. Authors refer some managerial irrationality models in context of studies of Roll (1986), Heaton (2002) and Malmendier and Tate (2001) in the same study.

Shefrin $(2005,2008)$ has indicated that some firms simply value financial flexibility and will issue debt so as to hold enough cash especially in times of uncertainty or if they foresee significant investments on the horizon. In such cases, they do not need the cash immediately or know whether they will need it but they may prefer to issue debt (and in some cases convertible debt) as a cheaper option to equity.

Ljungqvist and Wilhelm (2005) have investigated whether prospect theory can explain the behavior of managers in the IPO (Initial Public Offering) market. In their study they develop a scale which measures the satisfaction of who decided to IPO. The scale is based on work done by Loughran and Ritter. Scale assesses the decision-maker's choice among underwriters in subsequent securities offerings. They find that firms are significantly more likely to switch underwriters after the IPO when the behavioral factor points out that they were dissatisfied with the IPO underwriter's performance.

Barberis and Huang (2006) have examined equity puzzle. In their approach, most important factors are loss aversion and narrow framing. They study various ways of more traditional utility functions including narrow framing and loss aversion and indicate that this model features have an attractive way of thinking on the historical equity premium.

Dodonova and Khoroshilov (2006) have offered a definition for the use of stock option grants in managers' compensation. They indicates that in the case where the managers are loss averse, the optimal incentive contract must contain a substantial portion of stock options even when it should consist exclusively of stock grants for risk-averse managers.

Ben-David, Graham and Harvey (2007) have investigated overconfidence among American finance executives, and asked them to estimate 1 and 10 years of market returns and realized 80 percent confidence on their estimates. As a result, they have reached the finding that the overconfident managers value cash flows with low discount rates. In addition the overconfidence leads to use more debt, to invest more, to use more long term debt and to repurchase shares. And also the overconfident managers are less likely to pay dividends.

Devers, Wiseman and Holmes (2007) have argued that stock price volatility has positive effect on stock option value. They indicate, stock options compensation promote the managerial risk seeking. Thus, administrators and shareholders risk seeking and preferences will be harmonized.

Brown and Sarma (2007) have investigated the role of hubris and dominance behavior of managers in acquisition investments. As a result of the study they indicate that hubris (overconfidence) and dominance behaviors important factors in explaining of the acquisitions .

Campbell and Sharpe (2007) have tested the anchoring bias in their study. They examine whether expert consensus estimates of monthly economic bulletin from Money Market Services surveys from 1990-2006 have a tendency to be systematically biased toward the value of previous months' data bulletin. In conclusion, bond yields react only to the residual, or unpredictable, component of the surprise and not to the expected piece of the forecast error apparently induced by anchoring.

Fairchild (2009) has analyzed the managerial overconfidence in his study. Fairchild investigates this bias in terms of effects on financing decisions and firm value when investors face managerial moral hazard. The study examines the impact of combination of managerial overconfidence and moral hazard on the capital structure decisions. According to results, overconfidence can cause a decrease in debt, as the overconfident manager overestimates future investment opportunities, and hence reduces debt, compared to the rational manager, in order to invest in these new projects.

$\mathrm{Li}$ (2010) have examined the managers' self-serving attribution (SAB) bias. Li indicates that self-serving bias is a result of overconfidence bias. Having self-serving bias leads to higher investment-cash flow sensitivity and experience more negative market reactions around acquisition announcements. According to Li, self-serving bias also leads to tend having higher leverage, rely more on long-term debt financing. As a result managers commonly have self-serving attribution bias and this bias has implications for corporate policies.

Galasso and Simcoe (2011) have studied the impact of overconfidence on innovation decisions. For this purpose they have benefited from the scale that laid down by Malmendier and Tate. According to the findings in the companies which ones managed by overconfident managers, patents, citations, and research and development expenditures increases. And also they find that CEO overconfidence has a greater impact on innovation under intense product market competition. As a result their findings suggest that overconfident CEOs are more likely to take their firms in a new technology direction (Goldfarb et. al, 2012). 
Huang and Padmanabhan (2011) develop one and two period models to simulate and examine the impact of managerial anchoring on firm value. Authors have stated that if managers trust more their own estimates (this situation is managerial anchoring) than realized estimates, this can affect negatively to firm value. As a result of their study they indicate that the impact of managerial anchoring decreases if other costs associated with off-shoring increase their impact on firm value.

Libby and Rennekamp (2011) have also examined the managers' self-serving attribution bias and overconfidence. They state that in their experiment participants engage in self-serving attribution, giving greater weight to internal than external factors as explanations for good performance. This situation increases confidence in improved future performance, which increases their willingness to issue forecasts. Their study suggests that experienced financial manager survey participants believe other managers are likely to overestimate the extent to which they contribute to positive firm performance.

Baker, Pan and Wurgler (2012) have studied the effect of target company's past peak stock prices (reference point) on the acquisition and merger activities. According to the results of the study the last peak stock prices the was significant in explaining a variety of situations, such as offer prices and offer announcements effects.

Ammann, Ising and Kessler (2012) have considered the managerial disposition effect. They found strong evidence about the presence of disposition effect among mutual fund managers. In this context who have a lower disposition effect invest more in larger equities with a higher trade volume and have a higher past performance and higher risk-adjusted performance. And also they have found that the impact of the disposition effect on fund performance is limited.

Jackson, Keune and Salzsieder (2012) have studied if debt financing, relative to equity financing, causes managers to make non-value maximizing capital investment decisions. Their results reveal a strong debt-induced proclivity to invest additional resources in the asset, which suggests that debt financing may also induce overinvestment behavior in certain situations.

Andriosopoulos, Andriosopoulos and Hoque (2013) in their study, have discussed the repurchase of shares in terms of behavioral factors. In study, they have investigated influence of overconfidence and disclosure information on the completion rate of stock repurchases. They have found a strong relationship between overconfidence, information disclosure and completion rates of repurchase of shares.

Deshmukh, Goel and Howe (2013) examined the interaction between overconfidence and dividend policy. This interaction is expressed by a model. According to their findings, overconfidence bias leads to view external financing as costly. This perception builds financial slack for future investment needs by decreasing the current dividend payout. They also have reached the conclusion that the managers who show tend of overconfidence pay significantly low dividend compared to other managers.

Cen, Hilary and Wei (2013) have indicated that anchoring bias have significant effects on financial markets. They have tested the effect of anchoring bias, optimistic and pessimistic forecasts on estimated earnings per share, earnings surprises, stock splits and stock returns. Their results show that analysts make optimistic (pessimistic) forecasts when a firm's forecasts of earnings per share is lower (higher) than the industry median. And also firms with forecasts of earnings per share greater (lower) than the industry median experience extremely high (low) future stock returns.

Adam, Burg, Scheinert and Streitz (2014) have examined managerial optimism in context of the effect on the inclusion of performance pricing provisions in syndicated loan contracts (PSD). According to authors, optimism may cause managers to view PSD financing is a best and cheap way relative to other alternatives. They find that optimistic managers are more likely to issue PSD. Optimistic managers choose contracts with greater performance pricing sensitivity than rational managers. Their results show that behavioral factors can affect contract design for syndicated loans.

Blanc and Setzer (2015) have investigated the debiasing judgmental corporate cash flow forecasts. They indicate that the regression biases exist for all business divisions of the company. This correction increases forecast accuracy significantly. They state, statistical techniques can be used to minimize the effects of bias and thus more accurate predictions can be made.

Hribar and Yang (2015) have explored overconfidence bias in terms of effects on the management forecasts. They have reached the evidence that managerial overconfidence shows itself as excessive optimism in the cases of estimating future. The researchers have indicated that overconfidence leads managers to make and apply their own forecasts but commonly they make incorrect forecasts.

Adam, Fernando and Golubeva (2015) have examined the managerial overconfidence in terms of whether to help to explore the observed differences among corporate risk management practices. According to the results of the study, the effect of overconfidence on corporate risk management decisions is significant.

In Turkey, the studies about the subject of behavioral biases and their effects on financial decisions are limited. There are basically four studies. Two of them was conducted by Tomak $(2009,2013)$ who was investigated the biases in terms of entrepreneurs and the impact of overconfidence on the capital structure decisions. The other one was made by Kara (2011) who investigated anchoring bias in terms of corporate profits. And last one was made by Tekin (2015) as a dissertation. Tekin examines the effect of behavioral biases on firm financial decisions in his study.

\section{CONCLUSION AND DISCUSSION}

Behavioral finance takes the psychology science and uses it to explain the financial behaviors of the market participants and while doing this, examines the effects of several behavioral and psychological phenomenons on decision making. 
Behavioral finance draws more attentions of researchers on itself while adjusting the traditional finance theory assumptions that based on expected utility theory with hypotheses that based on prospect theory and real people behaviors which consisted of bias, emotion and heuristic.

Behavioral biases' effects are wide and all of the market participants like investors, corporations, managers, agencies etc. are exposed. Each manager commonly tend to overestimate their activities and achievements. No matter that he works in which company or sector. They have behavioral biases like overconfidence, framing, mental accounting, loss aversion, anchoring etc. in particular financial indicators such as earned income, revenues, cash flows (Mihalea and Claudia, 2014). Firm managers' behavioral biases not only effect to themselves and also it effects to the whole corporation. However, financial policies are influenced by behavioral biases.

According to Busenitz and Barney (1997) if the environment is not certain and complex, biases and heuristics can be an effective and efficient aim to decision making. In such cases, making wide and careful decisions are not possible. Therefore, investigation of the effect of behavioral and psychological biases on the decision making processes, present an effective way to estimate the appropriate decisions.

This paper presents a literature review of the managerial biases that affect to the corporate decisions. It depicts a general picture of behavioral finance and behavioral corporate finance applications in the field. This study, although not cover all of the behavioral finance studies in the literature, is thought it represents a whole and contains the most important works in the field.

As mentioned above managers can make decisions irrationally under the effect of certain biases. By means of studies in the literature, these biases which are subject to this study are as follows; overconfidence, loss aversion, anchoring, selfserving attribution, optimism, mental accounting, narrow framing, conservatism and disposition effect. Of course there are other biases like illusion of control, familiarity, sunk cost etc. that affect to individuals' specifically managers' and investors decision making processes. In this study we just tried to draw a broad framework as possible by focusing on the most mentioned biases in the literature.

The corporate and manager financial decisions that elaborated in this study are summarized as follows; takeover activity, benefits and costs of free cash flow, sensitivity of investment to cash flow, firm's social responsiveness, to avoid earnings decreases and losses, entrepreneurs imitate and explore, involved with trader, aggregate stock market, cross-section of average returns, IPO market, capital expenditures, mergers and acquisitions, stock option use, equity return, stock price volatility on stock option value, bond yield, earnings forecasting and earning management, asset pricing, firm value, corporate leverage rate, share buyback and dividend policy.

This study reveals the role of behavioral biases on the financial decisions taken in the company with the studies that are in the literature. It is recommended for the future studies, especially to be held in Turkey, the proposed findings in this study may be supported by empirical evidence. Therefore, it is hoped to be seminal and good source of this work for further studies especially which will be held in Turkey.

\section{REFERENCES}

1. Adam, T. R., Fernando, C. S., \& Golubeva, E. (2015). Managerial overconfidence and corporate risk management. Journal of Banking \& Finance, 60, 195-208.

2. Adam, T., Burg, V., Scheinert, T. \& Streitz, D., (2014), Managerial Optimism and Debt Contract Design: The Case of Syndicated Loans. Available at SSRN: http://dx.doi.org/10.2139/ssrn.2075955

3. Ammann, M., Ising, A., \& Kessler, S. (2012). Disposition effect and mutual fund performance. Applied Financial Economics, 22(1), 1-19.

4. Andriosopoulos, D., Andriosopoulos, K., \& Hoque, H. (2013). Information disclosure, CEO overconfidence, and share buyback completion rates. Journal of Banking \& Finance, 37(12), 5486-5499.

5. Baker, M., \& Wurgler, J. (2007). Investor Sentiment in the Stock Market. Journal of Economic Perspectives, 21(2), 129-152.

6. Baker, M., Pan, X., \& Wurgler, J. (2012). The effect of reference point prices on mergers and acquisitions. Journal of Financial Economics, 106(1), 49-71.

7. Barberis, N. \& M. Huang (2004), "Preferences with Frames: A New Utility Specification that Allows for the Framing of Risks,"Working paper, Yale University

8. Barberis, N., \& Huang, M. (2006). The loss aversion/narrow framing approach to the equity premium puzzle (No. w12378). National Bureau of Economic Research.

9. Barberis, N., \& Thaler, R. (2003). A survey of behavioral finance. Handbook of the Economics of Finance, 1, 10531128.

10. Bashir, T., Scholar, M. S., Rasheed, U., Raftar, M. S. S., Fatima, M. S. S., \& Maqsood, S. M. Impact of Behavioral Biases on Investors Decision Making: Male Vs Female. Journal of Business and Management, V. 10, I. 3 (May.-Jun. 2013), PP 60-68

11. Ben-David, I., Graham, J. R., \& Harvey, C. R. (2007). Managerial overconfidence and corporate policies (No. w13711). National Bureau of Economic Research.

12. Bernardo, A. E., \& Welch, I. (2001). On the evolution of overconfidence and entrepreneurs. Journal of Economics \& Management Strategy, 10(3), 301-330.

13. Blanc, S. M., \& Setzer, T. (2015). Analytical debiasing of corporate cash flow forecasts. European Journal of Operational Research, 243(3), 1004-1015.

14. Brown, R., \& Sarma, N. (2007). CEO overconfidence, CEO dominance and corporate acquisitions. Journal of Economics and business, 59(5), 358-379.

15. Burgstahler, D., \& Dichev, I. (1997). Earnings management to avoid earnings decreases and losses. Journal of accounting and economics, 24(1), 99-126. 
16. Busenitz, L. W., \& Barney, J. B. (1997). Differences between entrepreneurs and managers in large organizations: Biases and heuristics in strategic decision-making. Journal of business venturing, 12(1), 9-30.

17. Camerer, C. F. (2004). Prospect theory in the wild: Evidence from the field. Colin F. Camerer, George Loewenstein, and Matthew. Rabin, eds., Advances in Behavioral Economics, 148-161.

18. Campbell, S. D., \& Sharpe, S. A. (2009). Anchoring bias in consensus forecasts and its effect on market prices. Journal of Financial and Quantitative Analysis, 44(02), 369-390.

19. Cen, L., Hilary, G., \& Wei, K. C. (2013). The role of anchoring bias in the equity market: Evidence from analysts earnings forecasts and stock returns. Journal of Financial and Quantitative Analysis, 48(01), 47-76.

20. Cronqvist, H., Makhija, A. K., \& Yonker, S. E. (2012). Behavioral consistency in corporate finance: CEO personal and corporate leverage. Journal of financial economics, 103(1), 20-40.

21. Deshmukh, S., Goel, A. M., \& Howe, K. M. (2013). CEO overconfidence and dividend policy. Journal of Financial Intermediation, 22(3), 440-463.

22. Devers, C. E., Wiseman, R. M., \& Holmes, R. M. (2007). The effects of endowment and loss aversion in managerial stock option valuation. Academy of Management Journal, 50(1), 191-208.

23. Dodonova, A., \& Khoroshilov, Y. (2006). Jump bidding in takeover auctions. Economics Letters, 92(3), 339-341.

24. Fairchild, R. (2009). Managerial overconfidence, moral hazard problems, and excessive life cycle debt sensitivity. Investment Management and Financial Innovations, 6 (3).

25. Galasso, A., \& Simcoe, T. S. (2011). CEO overconfidence and innovation. Management Science, 57(8), $1469-1484$.

26. Goldfarb, A., Ho, T. H., Amaldoss, W., Brown, A. L., Chen, Y., Cui, T. H., ... \& Yang, B. (2012). Behavioral models of managerial decision-making. Marketing Letters, 23(2), 405-421.

27. Gökkurt, Ö. (1994). Enformetri, Bradford Yasası ve citation indeks. Türk Kütüphaneciliği, 8(1), 26-30.

28. Heaton, J. B. (2002). Managerial optimism and corporate finance. Financial management, 33-45.

29. Hribar, P., \& Yang, H. (2015). CEO overconfidence and management forecasting. Contemporary Accounting Research. $33(1)$,

30. Huang, C. H., \& Padmanabhan, P. (2011). Off-Shoring, Managerial Anchoring, and Firm Value: A Two Period Model. International Review of Business Research Papers, 7(5), 16-34.

31. Jackson, S. B., Keune, T. M., \& Salzsieder, L. (2013). Debt, equity, and capital investment. Journal of Accounting and Economics, 56(2), 291-310.

32. Kahneman, D. (2011). Don't blink! The hazards of confidence. The New York Times, 19.

33. Kahneman, D., \& Tversky, A. (1979). Prospect theory: An analysis of decision under risk. Econometrica: Journal of the Econometric Society, 263-291.

34. Kara, I., E., (2011). The Impact of Anchoring Bias on Corporate Profits and Shareholders Wealth, Journal of Economics and Finance Studies, 3(2), 105-115

35. Li, F. (2010), Managers' Self-Serving Attribution Bias and Corporate Financial Policies, Available at SSRN: http://ssrn.com/abstract=1639005 or http://dx.doi.org/10.2139/ssrn.1639005

36. Libby, R., \& Rennekamp, K. (2012). Self-Serving Attribution Bias, Overconfidence, and the Issuance of Management Forecasts. Journal of Accounting Research, 50(1), 197-231.

37. Ling Cen, Gilles Hilary \& K. C. John Wei (2013). The Role of Anchoring Bias in the Equity Market: Evidence from Analysts' Earnings Forecasts and Stock Returns. Journal of Financial and Quantitative Analysis, 48, pp 47-76. doi:10.1017/S0022109012000609.

38. Ljungqvist, A., \& Wilhelm, W. J. (2005). Does prospect theory explain IPO market behavior?. the Journal of Finance, 60(4), 1759-1790.

39. Malmendier, U., \& Tate, G. (2005). CEO overconfidence and corporate investment. The journal of finance, 60(6), 2661-2700.

40. Malmendier,U . \& G. Tate. (2001), "CEO Overconfidence and Corporate Investment,"Harvard University, Working Paper.

41. Mihaela, H. E. R. C. I. U., \& Claudia, O. G. R. E. A. N. (2014). Corporate Governance And Behavioral Finance: From Managerial Biases To Irrational Investors. Studies in Business and Economics, 9(1), 66-72.

42. Roll, R. (1986). The hubris hypothesis of corporate takeovers. Journal of business, 197-216.

43. Shefrin, H. (2001). Behavioral corporate finance. Journal of Applied Corporate Finance, 14(3), 113-126.

44. Shefrin, H. (2005) A Behavioral Approach to Asset Pricing. Boston: Elsevier Academic Press.

45. Sturdivant, F. D., Ginter, J. L., \& Sawyer, A. G. (1985). Managers' conservatism and corporate performance. Strategic Management Journal, 6(1), 17-38.

46. Tekin, B. (2015), The impact of behavioral biases on firms' financial decisions, PhD Dissertation, Sakarya University, Instute of Social Sciences, Sakarya, TURKEY.

47. Tomak, S. (2009). Girişimci hevristikleri: Bir kavramsal çözümleme. Eskişehir Osmangazi Üniversitesi Sosyal Bilimler Dergisi, 10(2).

48. Willman, P., Fenton-O'Creevy, M., Nicholson, N., \& Soane, E. (2002). Traders, managers and loss aversion in investment banking: a field study. Accounting, Organizations and Society, 27(1), 85-98.

49. Blanc, S. M., \& Setzer, T. (2015). Analytical debiasing of corporate cash flow forecasts. European Journal of Operational Research, 243(3), 1004-1015.

50. Hribar, P. and Yang, H. (2015), CEO Overconfidence and Management Forecasting. Contemporary Accounting Research, 33: 204-227. doi: 10.1111/1911-3846.12144 